\title{
Distinctive Morphological Features of a Subset of Cortical Neurons Grown in the Presence of Basal Forebrain Neurons In Vitro
}

\author{
Dun H. Ha, ${ }^{1}$ Richard T. Robertson, ${ }^{1}$ and John H. Weiss ${ }^{1,2,3}$ \\ Departments of ${ }^{1}$ Anatomy and Neurobiology, ${ }^{2}$ Neurology, and ${ }^{3}$ Psychobiology, University of California, Irvine, \\ Irvine, California 92697-4292
}

\begin{abstract}
Basal forebrain cholinergic neurons (BFCNs) provide the major subcortical source of cholinergic input to cerebral cortex and play an important role in regulating cortical activity. The present study examined the ability of BFCNs to influence neocortical neuronal growth by examining effects of the presence of BFCNs on certain cortical neurons grown under the controlled conditions of dissociated cell culture. Initial experiments demonstrated distinctive morphological features of a population of neurons (labeled with SMI-32, a monoclonal antibody to nonphosphorylated neurofilament proteins that labels pyramidal neurons in vivo) in cocultures containing basal forebrain (BF) and cortical cells. These neurons (large neurons immunoreactive for SMI-32 [SMI-32(+) neurons]) were characterized as having extensive axons, greater soma size, and more dendritic growth than did most SMI-32(+) neurons in the cultures. Stain-
\end{abstract}

ing for SMI-32 in cocultures in which the cortical neurons were labeled with a fluorescent marker before adding the BF cells indicated that virtually all large SMI-32(+) neurons were of cortical origin. Eliminating BFCNs with the selective cholinergic immunotoxin 192 lgG-saporin resulted in a > 80\% decrease in the number of large SMI-32(+) neurons, although causing little damage to other cells in the treated cultures; this suggests that survival or maintenance of large SMI-32(+) neurons may depend on ongoing trophic support from BFCNs. Thus, present findings suggest that BFCNs may provide powerful growthand/or survival-enhancing signals to a subset of cortical neurons.

Key words: ChAT; cholinergic; SMI-32; 192 IgG-saporin; pyramidal; culture; Alzheimer's disease; trophic interaction
Basal forebrain cholinergic neurons (BFCNs) project their axons to innervate neocortical neurons (Divac, 1975; Bigl et al., 1982; Eckenstein et al., 1988; Calarco and Roberston, 1995). In turn, neocortical neurons produce trophic factors, including the neurotrophins, that can bind to receptors at the cholinergic axon terminals and activate cellular processes supporting BFCN survival and differentiation. The trophic effects of the neurotrophins on BFCNs have been demonstrated during development (Hatanaka et al., 1988; Hsiang et al., 1989; Nonomura et al., 1995; Ha et al., 1996a) and also seem to extend into later life. For example, lesions of the fimbria-fornix, which disrupt retrograde transport of neurotrophins from the hippocampus to the basal forebrain (BF), result in atrophy of BFCNs in adult animals (Gage et al., 1986; Hefti, 1986; Sofroniew et al., 1993; Koliatsos et al., 1994). Conversely, elimination of BFCNs induces changes in neocortical neurons, including reductions in cell number, cell size, and dendritic length of some cortical neurons (Arendash et al., 1987; Hohmann et al., 1991; Wellman and Sengelaub, 1991, 1995; Roßner et al., 1995; Robertson et al., 1998). Collectively, these results indicate that BFCNs and cortical neurons are functionally interdependent and suggest that a breakdown in the function of one population may adversely effect the other. Such interdependence could be evidenced in degenerative conditions such as

\footnotetext{
Received Aug. 11, 1997; revised March 13, 1998; accepted March 13, 1998.

This work was supported by National Institutes of Health Grants NS 30884 (J.H.W.) and NS 30109 (R.T.R.) and by grants from the Alzheimer's disease and related disorders association (J.H.W.) and the Pew Scholars Program in the Biomedical Sciences (J.H.W.). We thank Janie Baratta, Kimberly Claytor, Mohsen Roshanaei, and Dr. Hong Z. Yin for technical assistance.

Correspondence should be addressed to Dr. John H. Weiss, Department of Neurology, University of California, Irvine, Irvine, CA 92697-4292.

Copyright (C) 1998 Society for Neuroscience $0270-6474 / 98 / 114201-15 \$ 05.00 / 0$
}

Alzheimer's disease (AD), in which both BFCNs and certain cortical pyramidal neurons degenerate (Davies and Maloney, 1976; Whitehouse et al., 1982; Morrison et al., 1987; Hof and Morrison, 1990; Hof et al., 1990; Cullen et al., 1997).

Numerous studies have examined the potential interdependence between BFCNs and cortical cells during development. For example, although BFCNs appear to survive in vivo in NGF knock-out mice (Crowley et al., 1994), experiments in vitro suggest that BFCNs require nerve growth factor or other cortically produced neurotrophic factors during a critical developmental period (Svendsen et al., 1994; Ha et al., 1996a). This critical period corresponds to the period of development of cortical innervation by the BFCNs in vivo (Dinopoulos et al., 1989; Calarco and Robertson, 1995). Whereas these and other studies have provided a general understanding of how cortical cells may regulate the development of BFCNs, our understanding of how BFCNs might modulate cortical development remains poor. Recent data, however, demonstrate that normal levels of cholinergic innervation may be important for development of dendritic features of some cortical pyramidal cells (Hohmann et al., 1991; Robertson et al., 1998).

In a previous study, we examined interactions between BFCNs and cortical neurons by growing dissociated BF and cortical cells together in cocultures. BFCNs in this highly simplified system form synapses with cortical neurons and display increased survival and enhancement of morphological features (Ha et al., 1996a). In the present study, a similar coculture system was used to examine effects of the presence of BFCNs on the phenotype of a subset of developing cortical neurons. Because BFCNs innervate cortical pyramidal neurons in vivo (Wainer et al., 1984; Houser et al., 1985; Houser, 1990) and elimination of the BFCNs 
seems to affect pyramidal target neurons, we set out to investigate effects of BFCNs on putative cortical pyramidal neurons in culture. As a neuronal marker, we chose the anti-nonphosphorylated neurofilament antibody SMI-32 that provides extensive morphological detail needed for these studies. In vivo, SMI-32 labels large subsets of pyramidal neurons, including those that are prone to degenerate in AD (Morrison et al., 1987; Hof and Morrison, 1990; Hof et al., 1990) and many of which express acetylcholinesterase $(\mathrm{AChE})$, suggesting that they may be important physiological targets of BFCN projections in cortex (Mesulam and Geula, 1991).

Parts of this paper have been published previously (Ha et al., 1996b).

\section{MATERIALS AND METHODS}

\section{Animals}

Brain tissues used for preparing cell cultures were derived from fetuses and neonates of timed-pregnant Sprague Dawley rats (Simonsen Labs). Pregnant dams were killed by lethal injections of sodium pentobarbital, and the fetuses were rapidly removed into cold medium; the neonates were killed by decapitation. All animals were deeply anesthetized with Halothane before death. The use of animals was conducted in accordance with the National Institutes of Health Guide for the Care and Use of Laboratory Animals.

\section{Cell culture preparation}

The general preparation and maintenance of cultures were performed primarily as described by Ha et al. (1996a). Briefly, the neocortex and the $\mathrm{BF}$ were dissected from the brains of fetuses (gestational age, 16-17 d) that were removed from timed-pregnant rats. The dissected cortex and $\mathrm{BF}$ were kept in separate dishes, minced, and incubated in trypsin for 30 min at $37^{\circ} \mathrm{C}$. Further dissociation of the brain tissues was accomplished by trituration using Pasteur pipettes with decreasing bore sizes. The resulting cell suspensions were diluted in a plating medium (PM) consisting of Eagle's minimal essential medium (MEM-Earle's salts, supplied glutamine-free) supplemented with $10 \%$ heat-inactivated horse serum, $10 \%$ fetal bovine serum, glutamine $(2 \mathrm{~mm})$, and glucose (total, 25 $\mathrm{mm}$ ). The diluted cell suspensions were plated on previously established monolayers of cortical astrocytes in 24-well tissue culture plates at $1.0-2.0 \times 10^{5}$ cells per $\mathrm{cm}^{2}$ for pure BF or pure cortical cultures and at $2.0-4.0 \times 10^{5}$ cells per $\mathrm{cm}^{2}$ for cocultures (see below) and were maintained at $37^{\circ} \mathrm{C}$ in a $5 \% \mathrm{CO}_{2}$ incubator. After $5-7 \mathrm{~d}$, cultures were treated with $10^{-5} \mathrm{M}$ cytosine arabinoside to reduce non-neuronal cell division. Cultures were fed with a maintenance medium that is similar to the PM but lacks fetal bovine serum. The astrocyte cultures used to plate neurons were prepared by plating cortical cells, taken from postnatal rats (day 1-3), directly on Falcon Primaria culture plates in medium supplemented with epidermal growth factor $(10 \mathrm{ng} / \mathrm{ml})$.

Several types of dissociated cell cultures were used in this study.

Pure BF or pure cortical cultures. For some experiments, pure BF and pure cortical cultures consisting of only BF and cortical cells, respectively, were prepared as described by Hartikka and Hefti (1988), Rose et al. (1993), and Ha et al. (1996a). Great care was taken to remove unwanted neighboring tissue to make these cultures as pure as possible. Although the BF dissection procedure may take much tissue from the medial septum/diagonal band, and less from the nucleus basalis magnocellularis itself, recent studies using organotypic basal forebrain slices cocultured with either neocortex or hippocampus demonstrate that the cholinergic neurons do not discriminate and that cells from either region of BF project equally well to both neocortex and hippocampus (Baratta et al., 1996).

Mixed BF-cortical cocultures. These were prepared by plating previously separated $\mathrm{BF}$ and cortical cells together. Two types of cocultures were prepared. The first consisted of BF and cortical cells that were combined, plated concurrently, and grown for a varied number of days (usually 18-20) depending on the experiment. When these cocultures were grown for only $5 \mathrm{~d}$, they are referred to as $5 \mathrm{CB}$ cocultures. In the second type of cocultures, the cortical cells were plated first and grown alone for 5 or $12 \mathrm{~d}$, after which BF cells were added for an additional $5 \mathrm{~d}$ (referred to as $5 \mathrm{C} 5 \mathrm{CB}$ and $12 \mathrm{C} 5 \mathrm{CB}$ cocultures, respectively). The converse setups, with the BF cells grown first for $5 \mathrm{~d}$ (5B5BC) or $12 \mathrm{~d}$
(12B5BC cocultures) followed by the addition of cortical cells, were also prepared.

Tandem cultures. To address issues regarding contact-mediated effects, we divided culture wells into two compartments using glass rings $(8 \mathrm{~mm}$; Bellco Glass, Vineland, NJ) coated with sterile vacuum grease. The dissociated BF cells were plated into the center compartment and allowed to attach. After 2-3 d, the rings were removed, and dissociated cortical cells were plated into the entire well. This resulted in tandem cultures that have regions consisting of only cortical cells as well as regions consisting of both cortical and $\mathrm{BF}$ cells but sharing a common astrocyte substratum and culture medium.

\section{CellTracker labeling}

Pure cortical cultures (12-d-old) were rinsed twice with a defined medium before the addition of the fluorescent cell marker 5-chloromethylfluorescein diacetate [CellTracker Green (CT); $10 \mu \mathrm{M}$; Molecular Probes, Eugene, OR]. The CT is taken up into cells in which it is de-esterified, producing stable intracellular fluorescence that can be detected with a fluorescein optical filter (excitation, $492 \mathrm{~nm}$; emission, $516 \mathrm{~nm}$ ). The cultures were incubated with the $\mathrm{CT}$ at $37^{\circ} \mathrm{C}$ until virtually all of the cortical neurons were clearly labeled (usually $1 \mathrm{hr}$ ), followed by removal of the $\mathrm{CT}$ with three media rinses. $\mathrm{BF}$ cells were then plated into these cultures and grown for $5 \mathrm{~d}$ as described above (12C5CB cocultures).

\section{Immunocytochemistry}

Cultures were fixed for $45 \mathrm{~min}$ in $4 \%$ paraformaldehyde at room temperature, followed by three PBS rinses. Cultures were then incubated in a blocking solution consisting of $10 \%$ horse serum in PBS for $1 \mathrm{hr}$ at $25^{\circ} \mathrm{C}$. Exposure to the appropriate primary antibodies was performed in fresh blocking solution for $24 \mathrm{hr}$ at $4^{\circ} \mathrm{C}$. Primary antibodies included SMI-32 (1:5000 in PBS with 0.2\% Triton X-100; made in mouse; Sternberger Monoclonals Inc., Baltimore, MA), anti-choline acetyltransferase (ChAT) (1:2000; made in goat; Chemicon, Temecula, CA), anti-p75 (1:5000; made in rabbit; Chemicon), or anti-GABA (1:10,000; made in rabbit; Sigma, St. Louis, MO). After the primary antibodies were removed with three PBS rinses, the cultures were incubated in secondary antibodies. For single staining, the appropriate biotinylated anti-mouse, anti-goat, or anti-rabbit secondary antibody was used $\left(1: 200 ; 1 \mathrm{hr} ; 25^{\circ} \mathrm{C}\right.$; Vector Laboratories, Burlingame, CA). After washout with PBS, avidinhorseradish peroxidase (ABC solution; Vector Laboratories) was added $\left(1 \mathrm{hr} ; 25^{\circ} \mathrm{C}\right)$, and labeled cells were visualized using 3-amino-9ethylcarbazole and $0.003 \% \mathrm{H}_{2} \mathrm{O}_{2}$ in acetate buffer $(50 \mathrm{~mm})$, $\mathrm{pH}$ 5.0. For double staining, cultures were first stained for ChAT, p75, or GABA using the procedure described above. After these stains were developed, the cultures were then incubated with the SMI-32 antibody (1:2500 in PBS with $0.2 \%$ Triton $\mathrm{X}-100 ; 24 \mathrm{hr} ; 4^{\circ} \mathrm{C}$ ). To avoid cross-reaction, we used an anti-mouse IgG-Cy3 secondary antibody (1:200; Jackson ImmunoResearch, West Grove, PA) to detect SMI-32 immunoreactive cells under fluorescent microscopy using a Cy3 optical filter (excitation, 510 $560 \mathrm{~nm}$; emission, $>590 \mathrm{~nm}$ ).

For stains of brain slices, two rat pups were killed at ages postnatal day 0 ( $\mathrm{P} 0), \mathrm{P} 7$, and $\mathrm{P} 14$, and tissue was fixed by perfusion with $4 \%$ parafor-

Table 1. Colocalization of ChAT or SMI-32 immunoreactivity with various other markers

$\operatorname{ChAT}(+)$
Large SMI-32(+)

\begin{tabular}{lcr}
\hline Neurons examined & 300 & 500 \\
$\mathrm{CT}(+) \#$ & 0 & 490 \\
$\mathrm{ChAT}(+) \#^{*}$ & - & 0 \\
$\mathrm{AChE}(+) \#^{*}$ & - & 0 \\
$\mathrm{GABA}(+) \#^{*}$ & - & 28 \\
p75 $(+)^{*}$ & 297 & 15 \\
$192 \mathrm{IgG}-\mathrm{Cy} 3(+)^{*}$ & 295 & 0 \\
\hline
\end{tabular}

For each double-labeling study, $300 \mathrm{ChAT}(+)$ or $500 \mathrm{SMI}-32(+)$ neurons (from five to six experiments) were randomly chosen and examined for colabeling with the other indicated markers. For studies examining ChAT, AChE, or GABA colabeling, large SMI-32(+) neurons were selected from both $12 \mathrm{C} 5 \mathrm{CB}$ cocultures $(240$ cells) and from BF-cortical cocultures grown for $\sim 18-20 \mathrm{~d}$ (260 cells); similar results were found in both culture types. A number sign indicates colabeling of $12 \mathrm{C} 5 \mathrm{CB}$ cocultures; an asterisk indicates colabeling of cocultures grown for 18-20 d. 

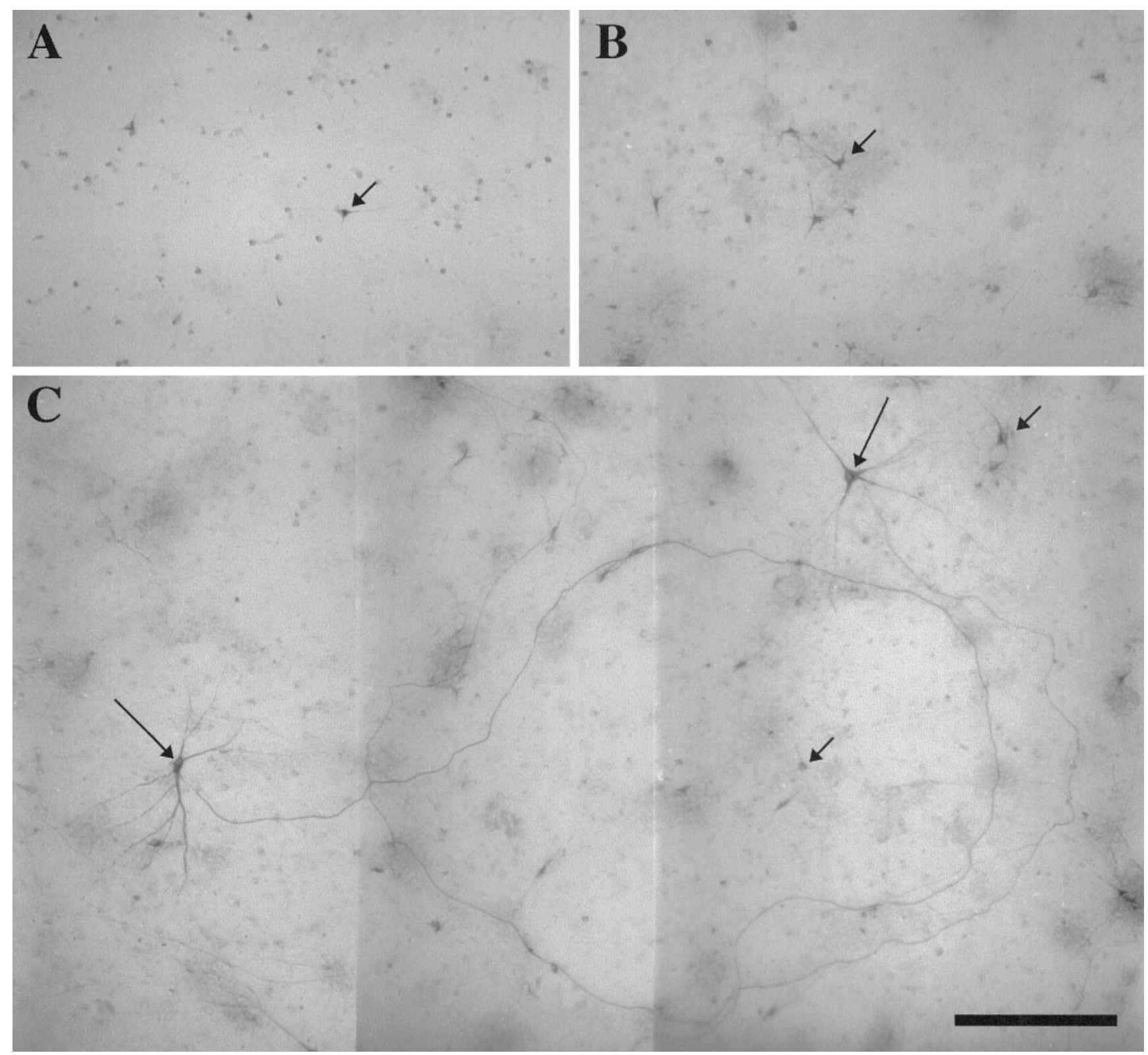

Figure 1. Morphological appearance of some SMI-32(+) neurons in cocultures. Photomicrographs show SMI-32-labeled neurons in a pure BF culture $(A)$, a pure cortical culture $(B)$, and a BF-cortical coculture $(C)$. The coculture consisted of both BF and cortical cells combined at the time of plating, and all three types of cultures were grown for 18-20 d before SMI-32 staining. Note the two large SMI-32(+) neurons (long arrows) and their long axons in the photomontage $(C)$. Short arrows indicate small SMI-32(+) neurons. Scale bar, $200 \mu \mathrm{m}$.

maldehyde. Frozen sections $(50 \mu \mathrm{m})$ were then processed for SMI-32 immunocytochemistry as described above.

\section{Acetylcholinesterase histochemistry}

AChE staining was performed as described by Tago et al. (1986) with minor modifications. Cultures were fixed with $4 \%$ paraformaldehyde for $\sim 40 \mathrm{~min}$, after which they were rinsed three times with $0.1 \mathrm{M}$ maleate buffer, $\mathrm{pH}$ 6.0. The cultures were then incubated in a fresh solution consisting of $300 \mu \mathrm{M}$ copper sulfate, $500 \mu \mathrm{M}$ sodium citrate, $50 \mu \mathrm{M}$ potassium ferricyanide, and $30 \mu \mathrm{M}$ acetylthiocholine iodide in $0.1 \mathrm{M}$ maleate buffer for $1-2 \mathrm{hr}$ in the dark. After being rinsed with PBS five times, the cultures were incubated in an intensification solution $(0.04 \%$ DAB, $0.3 \%$ nickel ammonium sulfate, and $0.003 \% \mathrm{H}_{2} \mathrm{O}_{2}$ in PBS) until cells were clearly stained (15-30 min). For AChE and SMI-32 double labeling, the AChE histochemistry was performed first, followed by SMI-32 immunocytochemistry. Again, an anti-mouse IgG-Cy3 second- ary antibody was used to visualize cells labeled with the SMI-32 primary antibody.

\section{IgG-saporin treatment}

Tandem cultures were prepared and maintained as described above. On day 12 and again on day 16 of a 22 d culturing period, some of the cultures were treated with the $192 \mathrm{IgG}$-saporin $(35-40 \mathrm{ng} / \mathrm{ml}$; Chemicon). The cultures were then stained with the SMI-32 antibody on day 22. In investigations of effects of $192 \mathrm{IgG}-$ saporin on BFCNs, pure BF cultures were grown for $14 \mathrm{~d}$ before treating with the $192 \mathrm{IgG}$-saporin for a varied number of days and staining for ChAT as described above. For experiments examining effects of $192 \mathrm{IgG}$-saporin treatment on pure $\mathrm{BF}$ cultures before addition of cortical cells, E17 BF cells were grown for 6-8 d before addition of the $192 \mathrm{IgG}-$ saporin for $3 \mathrm{~d}$. The cultures were then thoroughly washed, and E17 cortical cells were added for an additional $14 \mathrm{~d}$ before staining for ChAT or for SMI-32. 

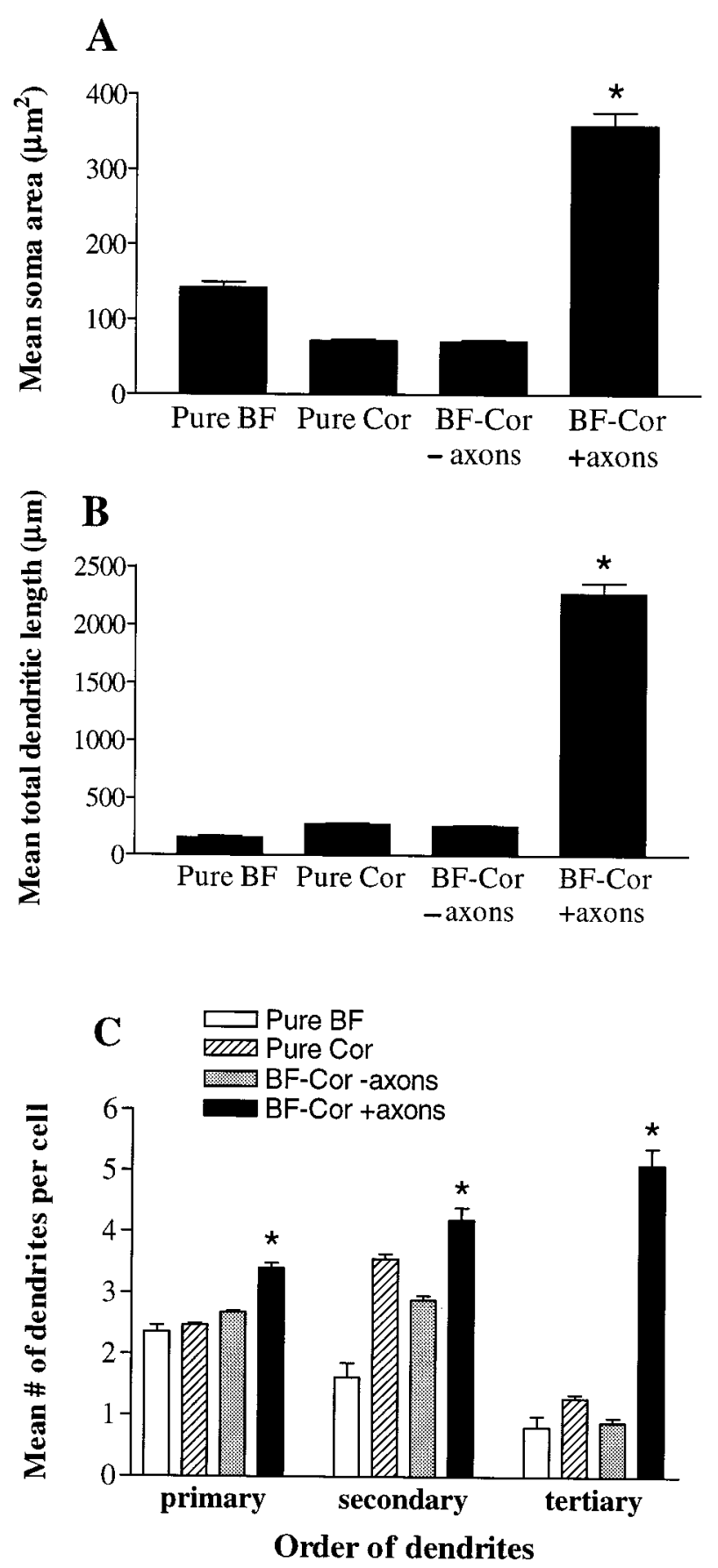

Figure 2. The morphological features of SMI-32(+) neurons in cocultures: a quantitative assessment. Fields from BF and cortical (Cor) cultures and from $\mathrm{BF}$-cortical cocultures such as those illustrated in Figure 1 were randomly selected and imaged, and all of the SMI-32(+) neurons within each field were measured for cell size $(A)$, extent of dendritic growth $(B)$, and extent of dendritic branching (indicated by the mean number of primary, secondary, and tertiary dendrites of all of the cells examined within each condition; $C$ ). In cocultures, the presence of a long $(>1500 \mu \mathrm{m})$ axon appeared to distinguish the minority $(\sim 5-10 \%)$ of SMI-32(+) neurons $(B F-C o r+a x o n s)$ that displayed enhanced morphological features from the remaining small SMI-32(+) neurons $(B F-C o r$ -axons) that were generally indistinguishable from those in pure cortical cultures. Values for neurons with such prominent axons are therefore graphed separately. Data are presented as the mean \pm SEM; $n=120$ fields for each condition, compiled from three experiments. An asterisk indicates a significant difference of the SMI-32(+) neurons with prominent axons from those lacking prominent axons in each culture condition; $p<0.001$ (ANOVA with Bonferroni post hoc test).
To study which cells in culture took up the $192 \mathrm{IgG}$-saporin, we conjugated the same $192 \mathrm{IgG}$ antibody to the fluorescent marker Cy3 (Chemicon) instead of saporin. Cocultures were treated with the 192 IgG-Cy3 (50-100 $\mathrm{ng} / \mathrm{ml})$ for 1-2 hr before fixation and staining with the SMI-32 antibody. In this case, the neurons immunoreactive for SMI-32 [SMI-32(+) neurons] were visualized with a secondary antibody labeled with the fluorescent marker Cy2 (seen with a fluorescein optical filter; 1:200; Jackson ImmunoResearch).

\section{Quantitative analysis}

For morphological measurements, stained cultures were viewed under bright-field microscopy $(100 \times)$, and fields were randomly selected by blindly scrolling through a grid pattern of approximately seven by seven fields and randomly stopping at intervals of three to four fields, such that 12-14 fields representing all areas of the dish and constituting $\sim 25 \%$ of total dish area were selected for imaging and analysis. A total of 30-40 fields were imaged from three to four sister cultures per condition for each experiment. The images were imported into a computer in which SMI-32(+) cells within each field were counted and their morphological features were measured using COMOS software from Bio-Rad (Hercules, CA). The parameters measured were cell area, total dendritic length, number of first-, second-, and third-order dendrites, and the presence or absence of a long $(>1500 \mu \mathrm{m})$ axon. Evaluation of data revealed that the presence of a long axon was the single trait that most reliably distinguished the large SMI-32(+) neurons from other SMI$32(+)$ neurons.

To assess double-labeling experiments, we first identified large SMI$32(+)$ neurons based on morphological criteria, including long axons, large soma size, and extensive dendritic arbor. Other experiments required the initial identification of ChAT $(+)$ neurons. These large SMI$32(+)$ or $\mathrm{ChAT}(+)$ neurons were then examined for the presence of label for other markers, as indicated in Table 1. For each double-labeling study, 500 large SMI-32(+) or $300 \mathrm{ChAT}(+)$ neurons from five to six experiments were examined.

The effects of $192 \mathrm{IgG}-$ saporin treatment were assessed by comparing cell counts of ChAT $(+)$ or SMI-32(+) neurons in treated and untreated cultures. In each culture well, the counts were determined from 52 consecutive, nonoverlapping microscope fields, covering over $95 \%$ of the well area, using low-power $(100 \times)$ bright-field optics. Neurons were considered $\mathrm{ChAT}(+)$ or SMI-32(+) if they were clearly stained and if at least two neurites could be identified. When the labeled cells displayed atrophic cell bodies, disrupted cell membranes, and broken processes, they were excluded from the counts. In each experiment, the percent loss of ChAT $(+)$ or SMI-32(+) neurons was calculated by comparing the mean number of intact stained cells in several (three to four) control (untreated) cultures with the mean number in several experimental (192 IgG-saporin-treated) cultures. In all experiments, both control and experimental conditions were on the same multiwell culture plate and derived from the same plating.

Values are given as the mean \pm SEM, normalized to control conditions in each experiment. Significance of the data was determined by ANOVA, with the Bonferroni post hoc test, using Instat software (Graph Pad, San Diego, CA).

\section{RESULTS}

\section{Large SMI-32(+) neurons are only found in BF-cortical cocultures}

Initial experiments were conducted to characterize the population of SMI-32(+) neurons in three different types of cultures (pure BF cultures, pure cortical cultures, and mixed BF-cortical cocultures) that were plated at gestational age $17 \mathrm{~d}$ (G-17) and grown for 18-20 d (see Materials and Methods). As illustrated in Figure 1, SMI-32 $(+)$ neurons were found in both pure $\mathrm{BF}(A)$ and pure cortical $(B)$ cultures; however, total numbers of SMI$32(+)$ neurons were $\sim 10$ times higher in the cortical cultures. The SMI-32(+) neurons in both of these pure culture types appeared relatively small, with short isodendritic neurites and no identifiable axons. In the combined BF-cortical cocultures, SMI-32(+) neurons were found with frequencies similar to those of the pure cortical culture but appeared as two morphologically distinct populations. The majority displayed morphological features sim- 


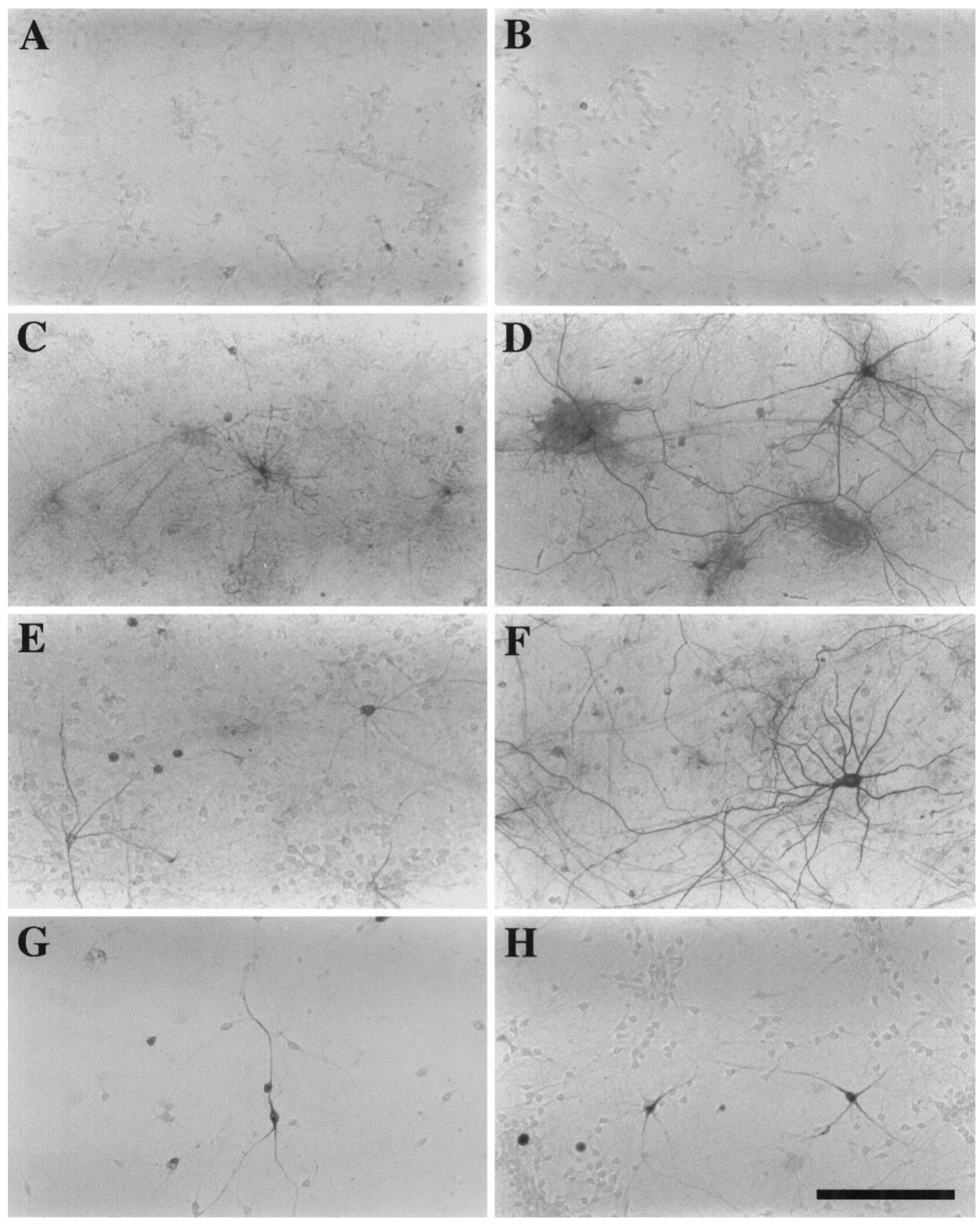

Figure 3. The appearance of large SMI-32(+) neurons in cocultures depends on the maturational state of the cortical neurons. Photomicrographs on the left show representative SMI-32-stained fields from pure cortical cultures grown for $5 \mathrm{~d}(A), 10 \mathrm{~d}(C)$, or $17 \mathrm{~d}(E)$ and from pure BF cultures grown for $17 \mathrm{~d}(G)$. Photomicrographs on the right show fields from cultures identical to those shown on the left except that either BF $(B, D, F)$ or cortical $(H)$ neurons were added for the last $5 \mathrm{~d}$ before staining. Note the presence of large SMI-32(+) neurons only in cocultures in which the cortical neurons were grown for at least $5 \mathrm{~d}$ before the addition of BF cells $(D, F)$. Scale bar, $100 \mu \mathrm{m}$. 

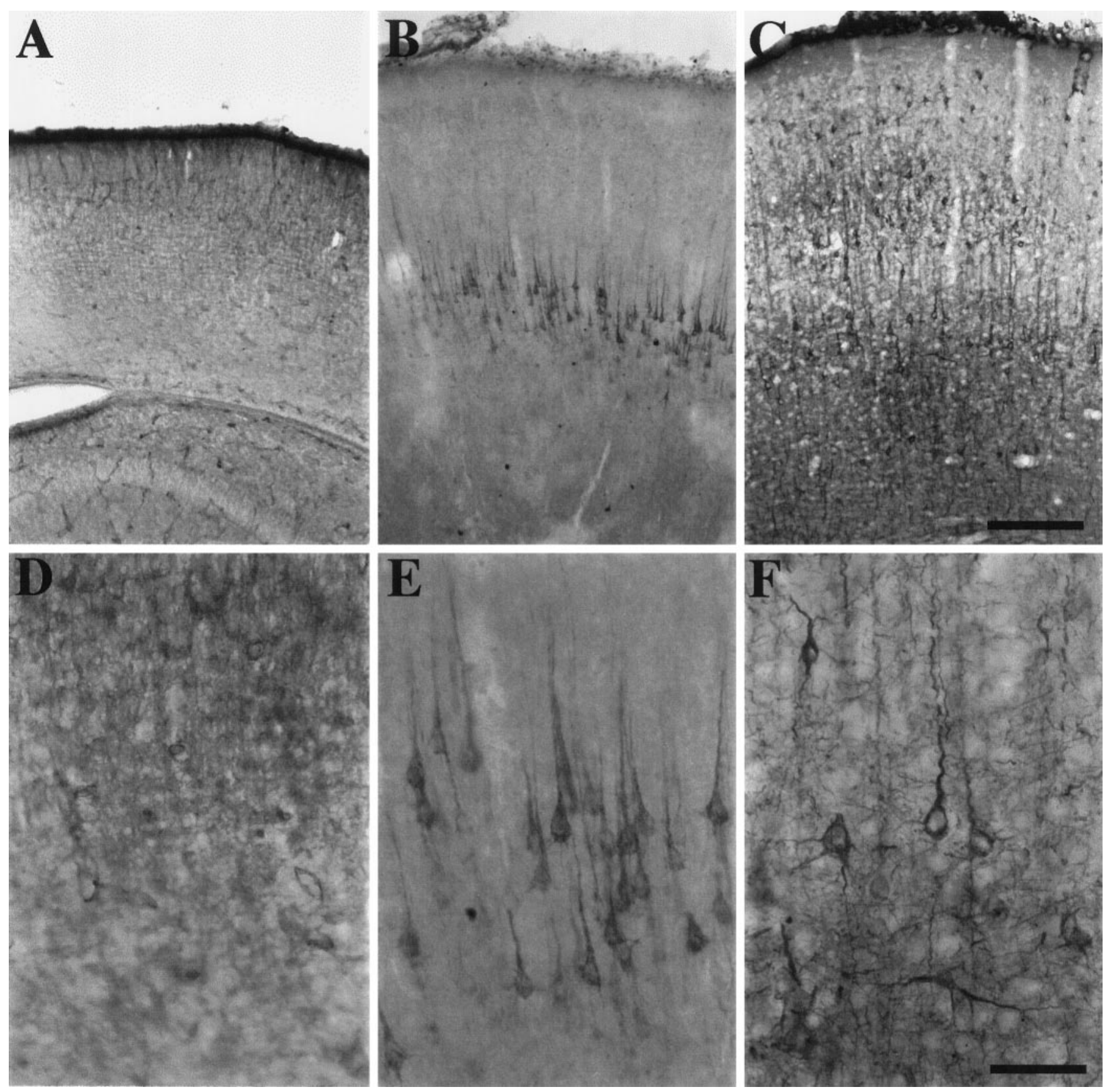

Figure 4. Patterns of SMI-32(+) neurons in developing neocortex. In animals killed at P0 $(A, D)$, no SMI-32(+) neurons are detected, although some labeling of capillaries can be seen. By P7 $(B, E)$, pyramidal neurons in layer V show prominent SMI-32 reactivity. By P14 $(C, F)$, pyramidal neurons predominantly in layers $\mathrm{V}$ and III are SMI-32(+). Scale bars: $A-C, 200 \mu \mathrm{m} ; D-F, 50 \mu \mathrm{m}$.

ilar to SMI-32(+) neurons found in pure cortical cultures [referred to as small SMI-32(+) neurons], whereas a minority [referred to as large SMI-32(+) neurons] displayed prominent axons that can often be traced for long distances as well as large cell bodies and extensive dendritic arbors (Fig. 1C).

Morphological characterization of SMI-32(+) neurons in randomly selected fields of the cocultures showed that SMI-32(+) neurons with long $(>1500 \mu \mathrm{m})$ axons [5-10\% of total SMI-32(+) neurons] also had substantially larger somata, longer dendrites, and greater dendritic branching than did most SMI-32(+) neurons (Fig. 2). Thus, the presence of long axons seemed to be a single criterion that could distinguish virtually all of the large from the small SMI-32(+) neurons.

\section{Phenotypic characteristics of SMI-32(+) neurons in cocultures is dependent on the age of cortical cells}

Initial experiments demonstrated the distinctive morphological features of a subset of SMI-32(+) neurons in BF-cortical cocultures grown for 18-20 d. Three sets of additional experiments were performed to examine whether the appearance of neurons with these morphological features was dependent on the age of either the cortical or the BF neurons. In the first set of experi- 

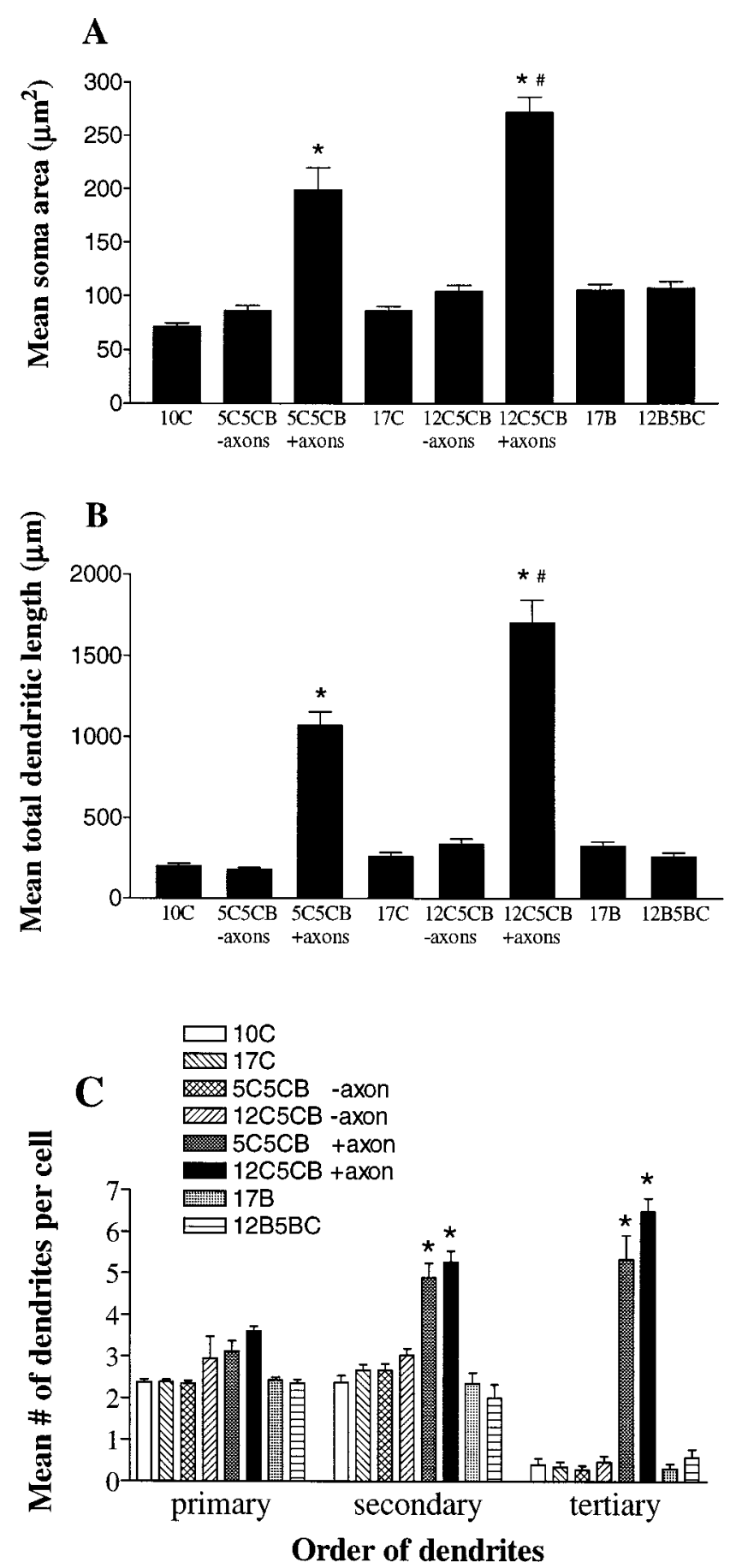

Figure 5. The appearance of large SMI-32(+) neurons in cocultures depends on the maturational state of the cortical neurons: a quantitative assessment. Fields from various BF, cortical, and coculture conditions were randomly selected and imaged, and all of the SMI-32(+) neurons within each field were measured for cell size $(A)$, extent of dendritic growth $(B)$, and extent of dendritic branching (indicated by the mean number of primary, secondary, and tertiary dendrites of all of the cells examined within each condition; $C$ ). Large SMI-32(+) neurons were only observed in cocultures in which cortical neurons had matured for either $5 \mathrm{~d}(5 C 5 C B)$ or $12 \mathrm{~d}(12 C 5 C B)$ before addition of BF cells for an additional $5 \mathrm{~d}$. Because the presence of a long $(>1500 \mu \mathrm{m})$ axon appeared to distinguish large SMI-32(+) neurons from small SMI-32(+) neurons, values for neurons with such prominent axons $(5 C 5 C B+$ axon; $12 C 5 C B$ +axon) are graphed separately; $n=90$ fields for each condition, compiled from three separate experiments. An asterisk indicates a significant difference of the SMI-32(+) neurons with prominent axons from those ments, G-17 cortical and BF cells were plated together concurrently and grown for only $5 \mathrm{~d}$ (5CB cocultures). No SMI-32(+) neurons were found in these cocultures or in sister control cultures containing only cortical (Fig. $3 A, B$ ) or BF cells (data not shown). This lack of staining in immature cortical neurons is consistent with the time course of appearance of SMI-32 immunoreactive neurons in vivo, in which distinct labeling of cortical neurons is primarily absent at birth and intensifies considerably by 2 weeks of age (Fig. 4).

The second set of experiments varied the number of days the cortical neurons were grown in culture before the addition of G-17 BF cells; the cocultures were then grown for 5 additional days before staining with the SMI-32 antibody. When cortical cultures were grown for either 5 or $12 \mathrm{~d}$ before the addition of BF cells (5C5CB or $12 \mathrm{C} 5 \mathrm{CB}$ cocultures), some SMI-32(+) neurons showed prominent axons and other features of large SMI-32(+) neurons (Fig. 3D,F). Control cortical cultures grown for 10 or $17 \mathrm{~d}$ without added BF cells contained only small SMI-32(+) neurons (Fig. 3C,E).

The third set of experiments used the converse coculture paradigm; G-17 cortical cells were added to previously established BF cultures and grown for 5 additional days. However, only small SMI-32(+) neurons were observed in these cultures (12B5BC cocultures) as well as in pure BF cultures grown for $17 \mathrm{~d}(17 \mathrm{~B}$ cultures) (Fig. 3G,H).

Quantitative analysis confirmed the above observations; large SMI-32(+) neurons were found only in the cortical cultures that had matured for 5 or $12 \mathrm{~d}$ before addition of BF cells (Fig. 5). Moreover, the morphological measures of large SMI-32 $(+)$ cells in $12 \mathrm{C} 5 \mathrm{CB}$ cocultures were greater than were those in $5 \mathrm{C} 5 \mathrm{CB}$ cocultures (Fig. 5). Thus, the rapid (within $5 \mathrm{~d}$ ) appearance of large SMI-32(+) neurons after addition of BF neurons depends on the maturational state of the cortical neurons.

\section{Large SMI-32(+) neurons are derived from cortex}

The observation that the rapid appearance of large SMI-32(+) neurons in cocultures depends on the degree of cortical neuronal maturation is consistent with the idea that the large SMI-32(+) neurons are of cortical origin. To determine more directly the origin of the large SMI-32(+) neurons, we prepared 12C5CB cocultures as described above but before the addition of BF cells, the 12-d-old cortical cells were labeled with the fluorescent marker CT. The CT labeled virtually all of the cortical cells in cultures and was readily detectable after $5 \mathrm{~d}$. Longer periods resulted in a significant loss of the labeling. Staining of these cocultures for SMI-32 revealed numerous large SMI-32(+) neurons, and of those examined, $98 \%$ were $\mathrm{CT}(+)$ (Fig. $6 A-C$; Table 1). In contrast, when these cocultures were stained for ChAT to label the BFCNs, none of the $300 \mathrm{ChAT}(+)$ neurons examined was $\mathrm{CT}(+)$ (Table 1$)$.

Additional double-labeling experiments, combining SMI-32 staining with ChAT, AChE, or GABA staining, were performed in an attempt to characterize further the identity of the large SMI-32(+) neurons. Two types of cocultures were used: BFcortical cocultures grown for $18-20 \mathrm{~d}$ and $12 \mathrm{C} 5 \mathrm{CB}$ cocultures. Nearly all $(>95 \%)$ of the large SMI-32(+) neurons in both types of cocultures lacked evidence of labeling for these markers (Fig.

lacking prominent axons in each culture condition, and a number sign indicates a significance difference of the condition $12 C 5 C B+$ axon from the condition $5 C 5 C B+$ axon; $p<0.01$ for all significant differences (ANOVA with Bonferroni test). 

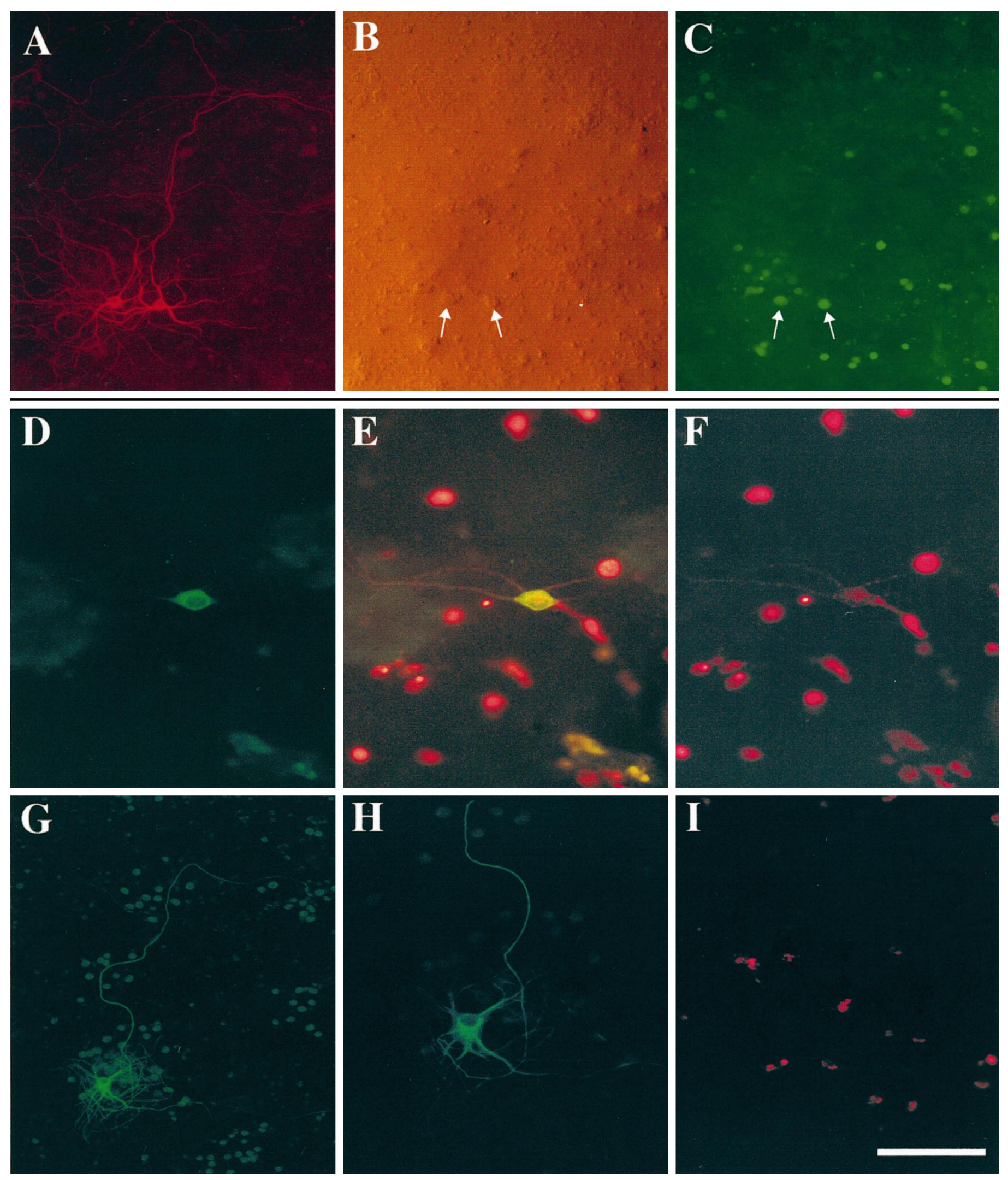

Figure 6. Double fluorescence colocalization of SMI-32 and ChAT immunoreativity with other markers. $A-C$, Large SMI-32(+) neurons are of cortical origin. Twelve-day-old cortical cultures were labeled with CT before addition of BF cells for an additional 5 d. Two large SMI-32(+) neurons are demonstrated under fluorescence ( $A$; using a Cy3-linked secondary antibody; red), under visible light $(B)$, and again under fluorescence to reveal the $\mathrm{CT}$ labeling $(C$; using a fluorescein optical filter; green). Note that the large SMI-32(+) neurons (arrows) are also labeled with the CT. D-I, Large SMI-32(+) neurons are not labeled by $192 \mathrm{IgG}-\mathrm{Cy} 3$. Photomicrographs show fields of BF-Cor cocultures that were grown for 18-20 d and then treated with 192 IgG-Cy3 hours before fixation and staining for ChAT $(D-F)$ or SMI-32 $(G-I)$. ChAT $(+)$ and SMI-32( + ) (Figure legend continues). 
7; Table 1). However, small patches of diffuse AChE staining were found on the cell bodies of numerous large SMI-32(+) neurons (Fig. 7E,F, insets).

\section{The appearance of large SMI-32(+) neurons in coculture requires close proximity between cortical and BF cells}

Dissociated tandem cultures (see Materials and Methods) were used to determine whether the appearance of large SMI-32(+) neurons in cocultures requires close proximity between cortical and BF cells. These tandem cultures contain both a coculture region (containing $\mathrm{BF}$ and cortical cells) and a pure-culture region (containing only cortical cells) that share the same medium and astrocyte substratum. In these tandem cultures, large SMI$32(+)$ neurons were found only in the coculture regions, whereas the small SMI-32(+) neurons were found in both pure and coculture regions (Fig. 8). Pure cortical cultures treated every $3 \mathrm{~d}$ for 14-20 d with conditioned medium taken from pure BF cultures contained only small SMI-32(+) neurons (data not shown). Further studies plated cultures containing the usual density of cortical neurons along with varied numbers of BF cells (the usual number, threefold lower, and threefold higher) to examine the "dose relationship" between numbers of BF cells and the number of large SMI-32 cells. These studies indicate a positive (although less than linear) relationship between numbers of BF cells and numbers of large SMI-32(+) cells under the experimental conditions with lower numbers of BF cells; under conditions with greater than usual numbers of $\mathrm{BF}$ cells, there appears to be a modest decrease in the number of large SMI-32(+) neurons (data not shown).

\section{Decreased numbers of large SMI-32(+) neurons after 192 IgG-saporin treatment}

The selective cholinergic immunotoxin 192 IgG-saporin was used in an attempt to determine whether BFCNs were responsible for inducing the distinctive features of large SMI-32(+) neurons. In initial control experiments, we found that treatment of pure BF cultures with 35-40 ng/ml $192 \mathrm{IgG}$-saporin for 3 or more days resulted in a loss of $>90 \%$ of $\mathrm{ChAT}(+)$ neurons, without causing evident death of other neurons (Fig. 9A). The effect of 192 IgG-saporin on SMI-32(+) neurons was examined in tandem cultures because they contain pure cortical culture regions (which provide an internal control for nonspecific toxicity) as well as $\mathrm{BF}$-cortical coculture regions in the same culture well. In untreated tandem cultures grown for $22 \mathrm{~d}$, the large SMI-32(+) neurons were found, as expected, only in the coculture regions (Fig. 10 $A$ ), whereas numerous small SMI-32(+) neurons were found in both the pure cortical and BF-Cor coculture regions. Treating tandem cultures with 35-40 ng/ml of 192 IgG-saporin on day 12 and again on day 16 of a $22 \mathrm{~d}$ culturing period resulted in a marked decrease in the number of large SMI-32(+) cells. Indeed, these cultures contained fragmented SMI-32-labeled neuritic processes, often lacking an intact cell body, that likely represent degenerated large SMI-32(+) neurons (Fig. 10B). Cell counts indicated a $>80 \%$ decrease in the numbers of large and an $\sim 30 \%$ decrease in the numbers of small SMI-32(+) neurons in the coculture regions, whereas numbers of small SMI-32(+) neurons in the pure cortical regions were unchanged (Fig. 9B).

Providing further support to the idea that the appearance of the large SMI-32(+) neurons in cocultures is specifically dependent on the presence of BFCNs, addition of 192 IgG-saporin to pure $\mathrm{BF}$ cultures before the addition of cortical cells markedly decreased numbers of both BFCNs and large SMI-32(+) neurons in the resultant cocultures (data not shown). However, the above observations do not eliminate the possibility that the loss of large SMI-32(+) neurons after addition of 192 IgG-saporin to cocultures could in part reflect direct effects of this immunotoxin. To address this issue, we first determined whether the large SMI$32(+)$ neurons express the p75 low-affinity NGF receptor, which is thought to be essential for mediating the endocytosis of the 192 IgG-saporin, by double staining cocultures for p75 and SMI-32. Examination of the double stains revealed that over $95 \%$ of the large SMI-32(+) neurons lacked immunoreactivity for p75 (Fig. $11 A-C$, Table 1$)$. In contrast, virtually all $\mathrm{ChAT}(+)$ neurons were strongly p75(+) (Fig. $11 D-F$, Table 1$)$.

To assess more directly the ability of different cells to internalize the 192 IgG-saporin, we produced a novel conjugate in which the $192 \mathrm{IgG}$ was linked to the fluorescent marker Cy3 instead of saporin. Virtually all $\mathrm{ChAT}(+)$ neurons in cocultures took up the 192 IgG-Cy3 reporter conjugate (Fig. $6 D-F$, Table 1 ). In contrast, none of the large SMI-32(+) neurons in sister cultures identically treated with the 192 IgG-Cy3 showed any Cy3 fluorescence, indicating little or no uptake (Fig. 6G-I, Table 1). Some astrocytes displayed $\mathrm{Cy} 3$ fluorescence (data not shown).

\section{DISCUSSION}

Beginning at late prenatal stages and continuing through the first few weeks of postnatal development, axons of BFCNs reach the cortex and establish the circuitry via which the cortical neurons can exert trophic effects on the BFCNs. This circuitry is also established between BFCNs and cortical neurons in dissociated cocultures; indeed, BFCNs exhibit extensive neuritic and somatic growth only when they are cocultured with cortical neurons (Ha et al., 1996a). Thus, both in vivo and in vitro studies have demonstrated trophic influences of cortical neurons on BFCNs. The purpose of the present study was to determine whether reciprocal effects exist, thus testing the hypothesis that BFCNs exert trophic influences on cortical neurons. Such effects have been suggested by in vivo studies showing cortical neuronal atrophy and loss and revealing alterations in dendritic morphology and differentiation of cortical neurons after placement of excitotoxic or electrolytic lesions in the BF (Arendash et al., 1987; Hohmann et al., 1991; Wellman and Sengelaub, 1991, 1995). Recently, a study using the specific cholinergic immunotoxin $192 \mathrm{IgG}$-saporin to selectively remove BFCNs in vivo demonstrated that reductions in cholinergic innervation result in significantly reduced branching of apical dendrites as well as reduced numbers of dendritic spines of pyramidal neurons in the visual cortex (Robertson et al., 1998). Because BFCNs innervate cortical pyramidal neurons in vivo (Wainer et al., 1984; Houser et al., 1985) and many of the sequelae observed after BF lesioning in vivo are expressed by pyramidal neurons, the present study focused on the subset of

neurons are viewed under fluorescence $(D, G, H)$ using a Cy2-linked secondary antibody and a fluorescein optical filter (green), with a double exposure showing both Cy2 and Cy3 fluorescence $(E)$, and with only a Cy3 optical filter to show 192 IgG-Cy3 accumulation $(F, I ; r e d)$. Note that the ChAT $(+)$ but not the SMI-32(+) neuron is clearly labeled with the $192 \mathrm{IgG}-\mathrm{Cy} 3$. The scattered areas of fluorescence in $F$ and $I$ are nonspecific clumps of stain and do not represent neurons. Scale bars: $A-C, G, 100 \mu \mathrm{m} ; D-F, H, I, 50 \mu \mathrm{m}$. 

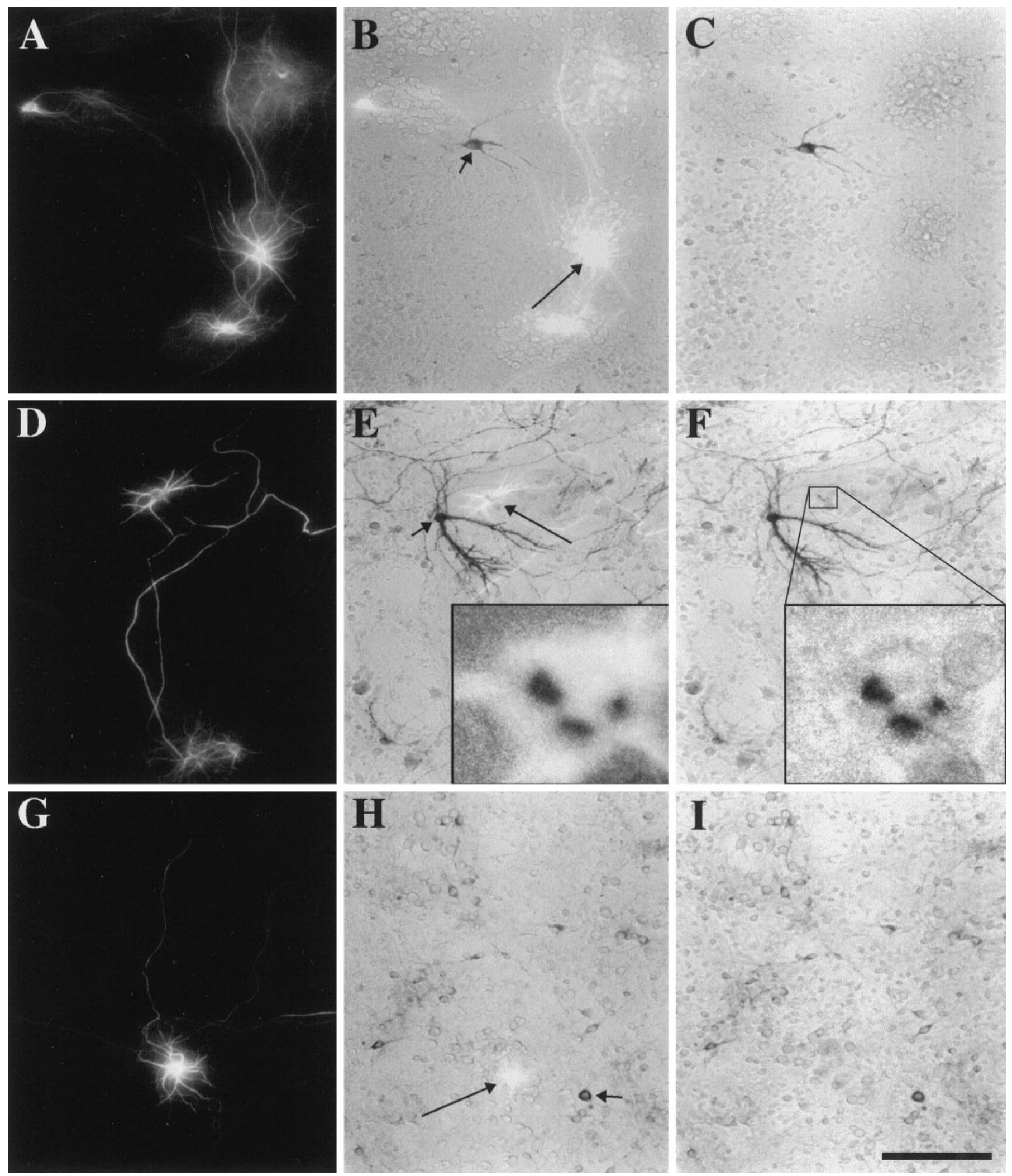

Figure 7. Large SMI-32(+) neurons are neither cholinergic nor GABAergic. Photomicrographs of cocultures (12C5CB) double stained for SMI-32 with ChAT $(A-C)$, AChE $(D-F)$, or GABA $(G-I)$ are shown. In each case, fields containing a large SMI-32 $(+)$ neuron are visualized under Cy3 fluorescence to reveal the SMI-32 stain $(A, D, G)$, under both bright field and fluorescence $(B, E, H)$, or under bright field $(C, F, I)$. Note that in all cases, the large SMI-32(+) neurons (long arrows $[B, E, H]$ ) show no labeling with the other markers examined, whereas other neurons in the same field (short arrows) are strongly $\mathrm{ChAT}(+)(C), \operatorname{AChE}(+)(F)$, or GABA $(+)(I)$. The insets in $E$ and $F$ show high magnification of two diff use patches of AChE reaction product on the cell body of the large SMI-32(+) neuron in that field (seen under fluorescence in $D, E$ ). Scale bar, $100 \mu \mathrm{m}$. 

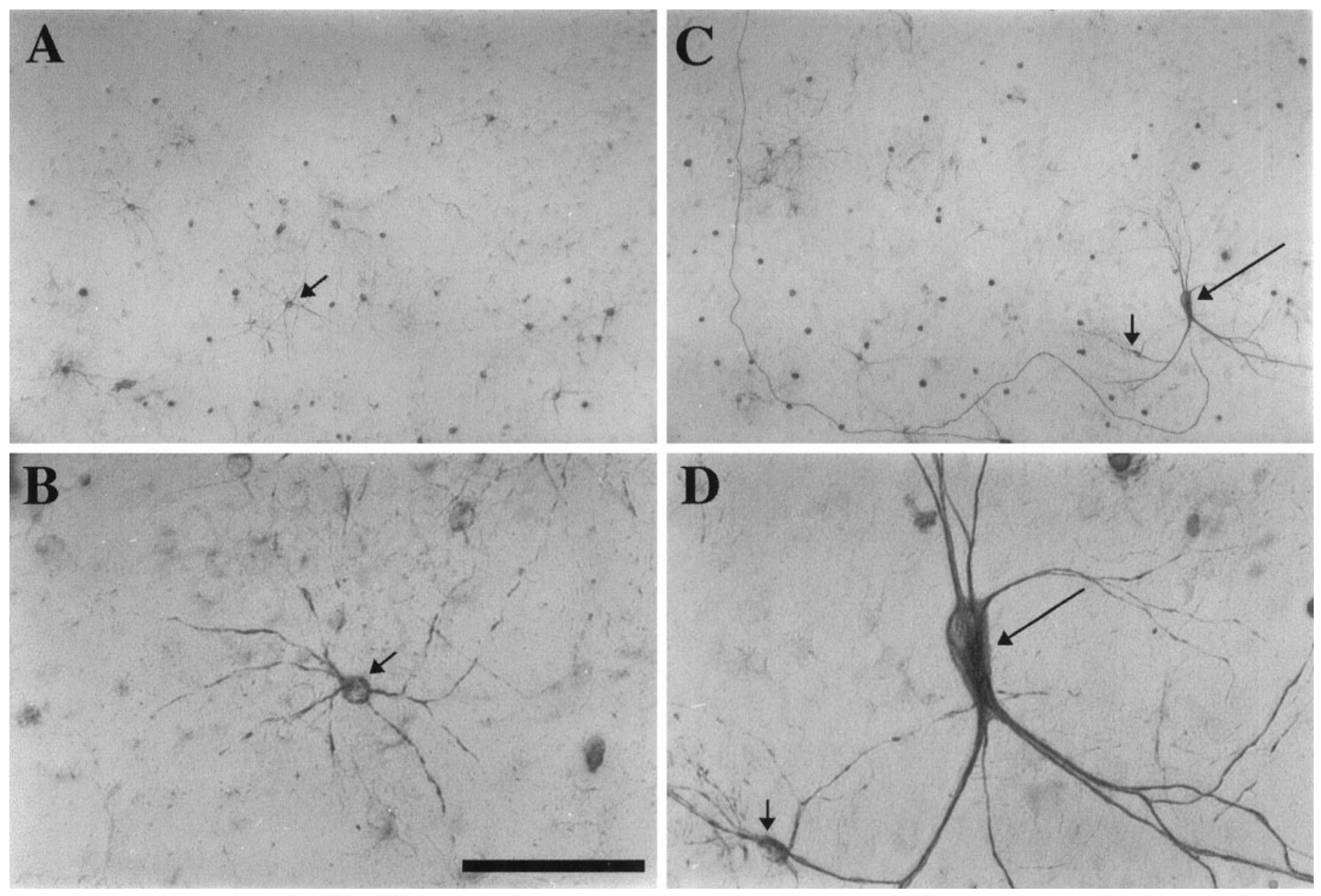

Figure 8. Large SMI-32(+) neurons are found only in coculture regions of tandem cultures. Photomicrographs show low and high magnification of representative SMI-32(+) neurons in the pure cortical $(A, B)$ and the coculture $(C, D)$ regions of a tandem culture. Note the large SMI-32(+) neuron (long arrows) in the coculture region and the small SMI-32(+) neurons (short arrows) in both pure and coculture regions of the tandem culture; no large SMI-32(+) neurons were present in the pure cortical regions of the cultures. Scale bars: $A, C, 200 \mu \mathrm{m} ; B, D, 50 \mu \mathrm{m}$.

neurons in culture labeled with the monoclonal antibody SMI-32 that labels subsets of cortical pyramidal neurons in vivo (Morrison et al., 1987; Hof and Morrison, 1990; Hof et al., 1990) (see also Fig. 4).

The primary finding of this study is that cocultures containing both BF and cortical cells display a subset of SMI-32immunoreactive neurons with distinctive morphological features: prominent axons, large somata, and long-branching dendrites. Thus, the appearance of these large SMI-32(+) neurons in cocultures is consistent with our working hypothesis that BFCNs may induce growth and/or increase survival of subsets of cortical pyramidal neurons in culture. Present data do not distinguish between the possibility that the appearance of the large SMI$32(+)$ neurons reflects morphological enhancement of small SMI-32(+) cells present in the cultures or induction of a new population of neurons that would either not survive or not be observed by SMI-32 staining in pure cortical cultures.

\section{Phenotypic identity and cortical origin of large SMI-32(+) neurons}

Several lines of evidence support the hypothesis that the large SMI-32(+) cells are a subset of cortical pyramidal neurons. First, studies using the fluorescent marker $\mathrm{CT}$ provided direct evidence that virtually all of the large SMI-32(+) neurons are derived from cortex and not from BF. Specificity of the CT labeling for cortical neurons was achieved by complete removal of the CT with media rinses before the addition of BF cells and also by the enzymatic cleavage of the CT, after it is inside the cell, to cell membraneimpermeable fluorescent products.

Second, the finding that large SMI-32(+) neurons do not express the enzymes ChAT or AChE is consistent with their presumed cortical origin and a noncholinergic identity. Interestingly, many of these neurons had small patches of AChE reaction product on their somata. This pattern of staining is entirely distinct from the extensive somatic and neuritic labeling shown by the BFCNs and is suggestive of a pyramidal identity, because many pyramidal neurons in neocortical layers III and V express AChE and are strongly SMI-32-immunoreactive (Mesulam and Geula, 1991). Further support for a pyramidal identity comes from studies demonstrating that large SMI-32(+) neurons are GABA-negative.

\section{Conditions under which large SMI-32(+) neurons are present}

As large SMI-32(+) neurons are only found in cocultures, expression of the distinctive morphological features of these neurons seems to be mediated via interactions between $\mathrm{BF}$ and cortical cells. In addition, the observation that destruction of most BFCNs in BF cultures by $192 \mathrm{IgG}$-saporin exposure before the addition of cortical cells results in markedly decreased numbers of 

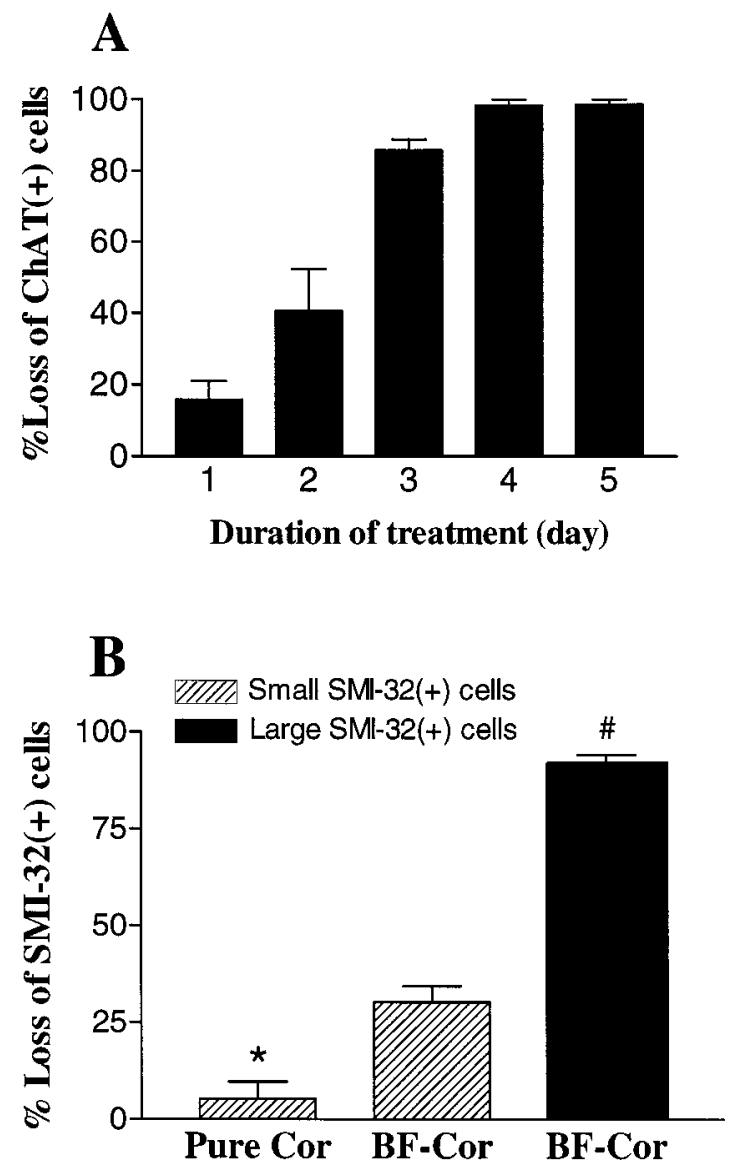

Figure 9. Loss of ChAT $(+)$ and large SMI-32(+) neurons in cultures treated with $192 \mathrm{IgG}-$ saporin. $A$, Fourteen-day-old pure BF cultures were treated with $192 \mathrm{IgG}$-saporin (35-40 ng/ml) for 1-5 d, followed by ChAT staining and evaluation of cell loss (by comparison with untreated sister cultures). Note the marked ChAT $(+)$ neuronal cell loss after 3 or more days of exposure to $192 \mathrm{IgG}$-saporin; $n=12$ cultures per condition from three experiments. $B$, Tandem cultures were treated with the $192 \mathrm{IgG}-$ saporin on day 12 and again on day 16, followed by SMI-32 staining on day 22. Cell loss was evaluated in relation to untreated sister cultures. Note the loss of most large SMI-32(+) neurons (present in the coculture or $B F-C o r$ regions of the tandem cultures), the minimal loss of small SMI-32(+) neurons in pure cortical regions, and the partial loss of small SMI-32(+) neurons in the coculture regions; $n=9$ cultures per condition, compiled from three experiments. An asterisk indicates significant differences from $\mathrm{BF}-\mathrm{Cor}$ cocultures, and a number sign indicates significant differences from small SMI-32(+) neurons; $p<0.001$ for all significant differences (ANOVA with Bonferroni test).

large SMI-32(+) neurons suggests that the growth- and/or survival-stimulating factors depend specifically on the presence of BFCNs. The further observation that large SMI-32(+) neurons were only present in the coculture regions of tandem cultures indicates that close proximity between BFCNs (or their axonal processes) and cortical cells may be necessary for any growth- or survival-stimulating signals to be transmitted. Thus, the effects could be mediated by direct interactions between BFCNs and SMI-32(+) neurons, as through synaptically transmitted signals, or by actions of cell surface proteins. Alternatively, soluble factors that are effective only over short distances could be produced. Although the suggestion that BFCNs may influence SMI$32(+)$ central neurons directly is an attractive one, at present we cannot eliminate the possibility that BFCNs affect an intermedi-
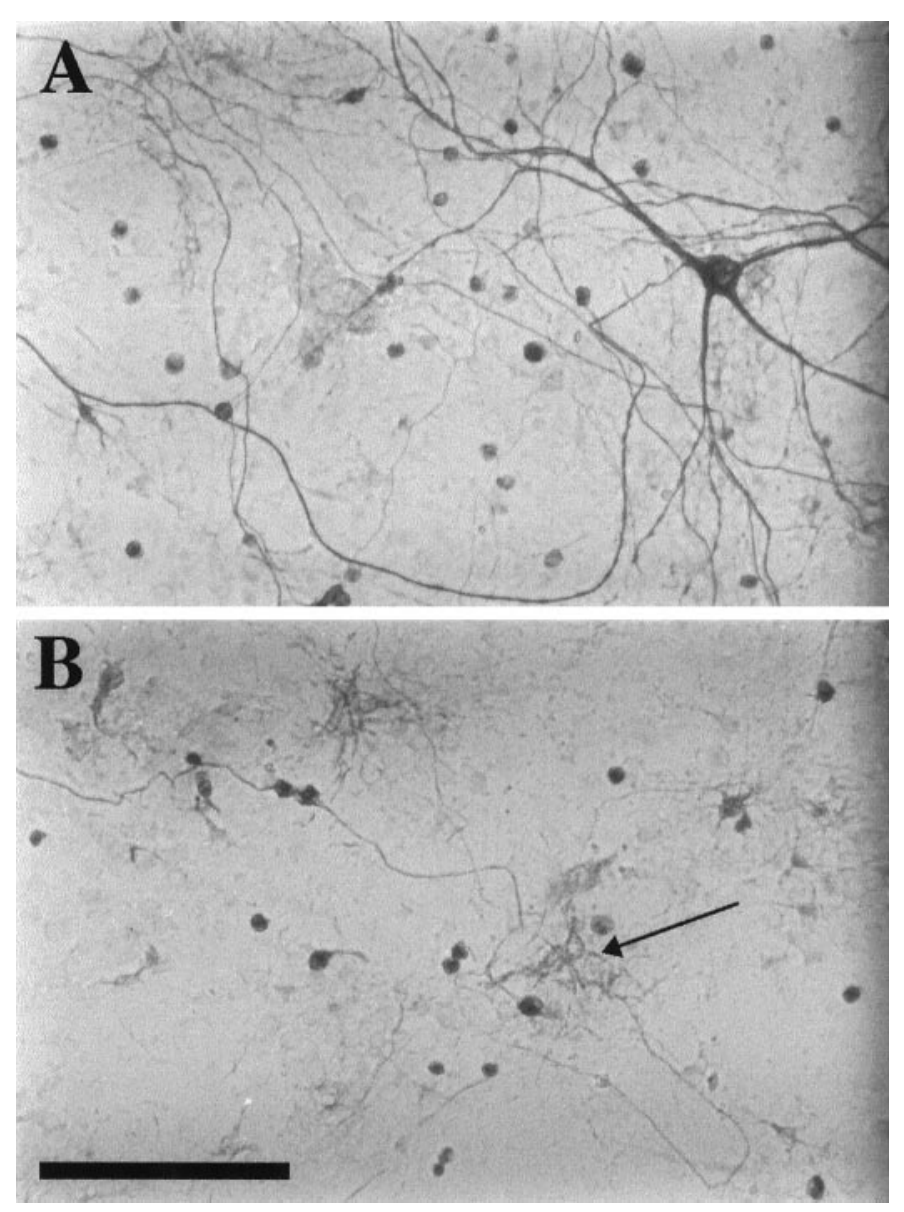

Figure 10. The immunotoxin $192 \mathrm{IgG}-$-saporin induces degeneration of large SMI-32(+) neurons. Tandem cultures were treated with the 192 IgG-saporin on day 12 and again on day 16 , followed by SMI-32 staining on day 22. A healthy large SMI-32(+) neuron is seen in the coculture region of an untreated tandem culture $(A)$, whereas in an immunotoxintreated sister culture $(B)$, fragmented SMI-32(+) neuronal processes are seen along with a faintly labeled and probably degenerating cell body (arrow). Scale bar, $100 \mu \mathrm{m}$.

ate population of cells, which in turn affect the SMI-32(+) neurons.

The morphological parameters of large SMI-32(+) neurons increased with increasing age of the cortical neurons, suggesting that the cortically derived large SMI-32(+) neurons become more responsive to BF-dependent factors with increasing maturation. The age in vitro at which cortical neurons appear to become responsive to the BF cells is $\sim 5-10 \mathrm{~d}$ in culture. This maturational level corresponds both to the time of appearance of SMI-32-immunoreactivity in cortical pyramidal neurons (see Fig. 4) and to the time at which cortical neurons are becoming differentiated and innervated by BFCNs in vivo (Dinopoulos et al., 1989; Gould et al., 1991; Calarco and Robertson, 1995; De Carlos et al., 1995).

\section{Maintenance of morphological features of large SMI-32(+) neurons by BFCNs}

Results from experiments using the cholinergic immunotoxin 192 IgG-saporin to eliminate BFCNs support the hypothesis that the presence of large SMI-32(+) neurons in cocultures requires the ongoing presence of BFCNs. Several studies have demonstrated the effectiveness and specificity with which 192 IgG-saporin kills 

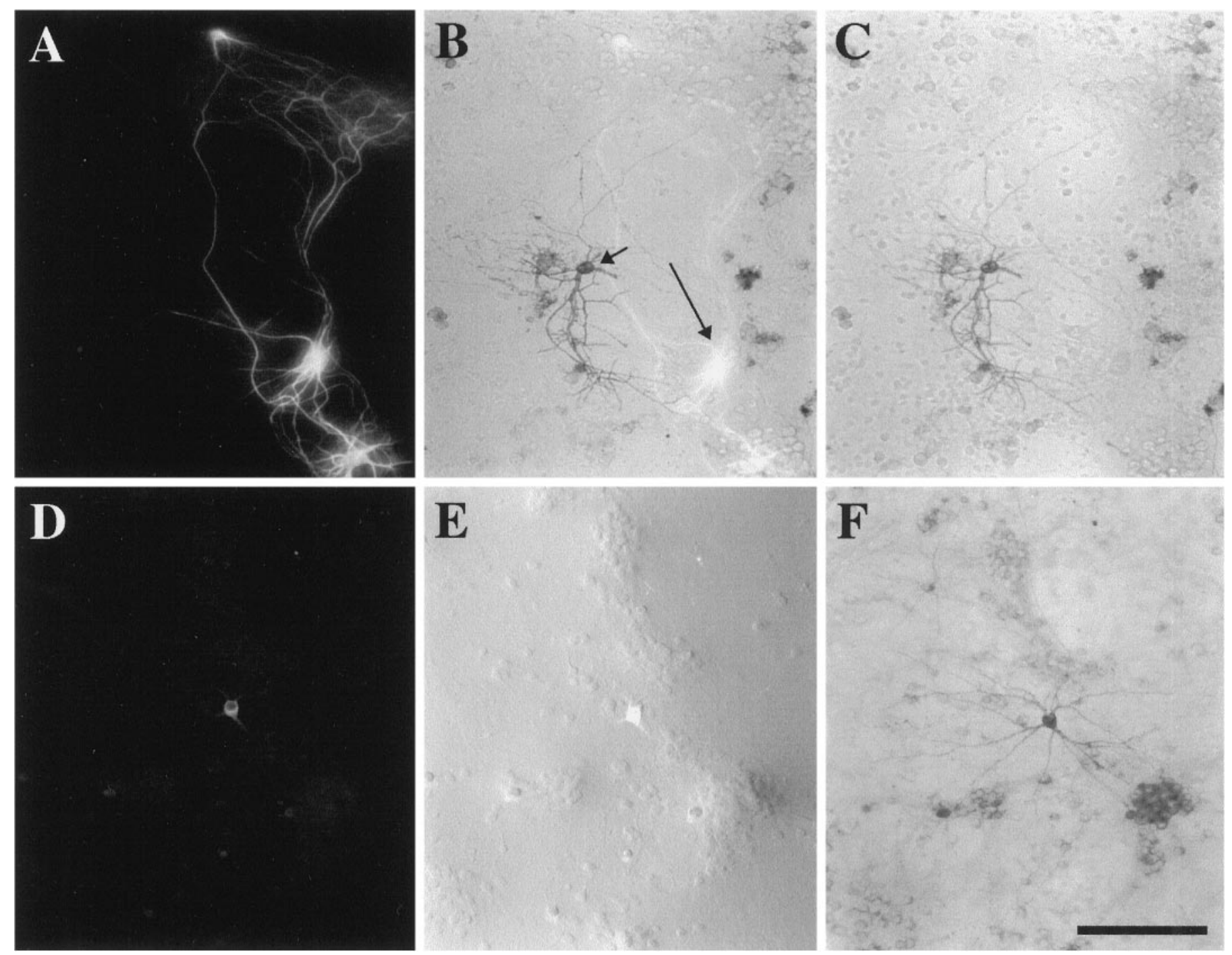

Figure 11. Large SMI-32(+) neurons do not express the p75 receptor. Photomicrographs show 18-d-old BF-Cor cocultures double stained for p75 and SMI-32 $(A-C)$ or ChAT $(D-F)$. Note that the large SMI-32(+) neurons $(A, B$; under fluorescence) lack p75 immunoreactivity $(B, C)$. The short arrow in $B$ indicates a p75(+) neuron in proximity to the large SMI-32(+) cell (long arrow). In contrast, the ChAT $(+)$ neuron $(D, E)$ is strongly p75(+) $(F)$. Scale bar, $100 \mu \mathrm{m}$.

the BFCNs in vivo (Wiley et al., 1991; Book et al., 1992, 1995; Heckers et al., 1994; Roßner et al., 1995). In cultures, the 192 IgG-saporin appears to have similar specificity; that is, treating cocultures with this immunotoxin resulted in near complete loss of the BFCNs, whereas the background cells did not appear to be affected. However, this treatment resulted in a significant reduction in the number of large SMI-32(+) neurons in the coculture regions of tandem cultures. If we accept for the moment that the primary effect of $192 \mathrm{IgG}$-saporin is on BFCNs (discussed in the next paragraph), the decrease in numbers of large SMI-32(+) neurons could reflect a secondary effect, either from a loss of growth stimulation or from degeneration or atrophy of large SMI-32(+) neurons that were already present after removal of BFCNs. Evidence of fragmented axons and damaged somata of large SMI-32(+) neurons suggests that the latter mechanism plays a role. These findings are compatible with in vivo studies showing various altered cortical phenotypes (Arendash et al., 1987; Hohmann et al., 1991; Wellman and Sengelaub, 1991, 1995) and specific structural abnormalities of developing pyramidal neurons (Robertson et al., 1998) after lesioning of the BFCNs.

The conclusion that the decrease in number of large SMI$32(+)$ neurons is secondary to $192 \mathrm{IgG}$-saporin-induced loss of BFCNs requires the demonstration that the toxic effects of the 192 IgG-saporin are highly selective. Although present results cannot completely eliminate possible direct toxic effects of 192 IgG-saporin on large SMI-32(+) neurons, three lines of experimental data support this contention. First, specificity of the 192 $\mathrm{IgG}$-saporin for BFCNs is dependent on the presence of the $\mathrm{p} 75$ low-affinity neurotrophin receptor, possibly in combination with the high affinity neurotrophin tyrosine kinase receptors, which are necessary for binding and internalization of the $192 \mathrm{IgG}-$ saporin (Chandler et al., 1984; Taniuchi and Johnson, 1985; Wiley et al., 1991; Gargano et al., 1997). Thus, the observation that all of the $\operatorname{ChAT}(+)$ neurons in culture were found to be $\mathrm{p} 75(+)$, whereas the large SMI-32(+) neurons were nearly all p75negative, would favor the selective ability of BFCNs to bind 
directly and take up the $192 \mathrm{IgG}$-saporin. Second, labeling of all of the ChAT $(+)$ cells, but none of the large SMI-32(+) neurons, with the $192 \mathrm{IgG}-\mathrm{Cy} 3$ provides more direct evidence that BFCNs, rather than large SMI-32(+) neurons, readily take up 192 IgG conjugates. The significance of the $192 \mathrm{IgG}-\mathrm{Cy} 3$ labeling of some astrocytes is uncertain, because in vivo studies have demonstrated mild or no astroglial reaction to $192 \mathrm{IgG}$-saporin treatment (Book et al., 1995; Roßner et al., 1995). Finally, the lack of degeneration of small SMI-32(+) neurons in the pure cortical regions of tandem cultures after 192 IgG-saporin treatment provides evidence against nonspecific toxic effects on the overall SMI-32(+) neuronal population. The loss of some small SMI$32(+)$ neurons only in coculture regions of treated tandem cultures could indicate the presence at the time of staining of some neurons that have been affected by the presence of the BFCNs, although not manifesting the distinctive morphological features of typical large SMI-32(+) neurons.

\section{Conclusions}

The present data suggest that BFCNs induce powerful survivaland/or growth-enhancing effects on a distinct subpopulation of cortical neurons. Furthermore, selective destruction of BFCNs seems to result in a secondary loss or atrophy of these cortical neurons. These observations are consistent with several in vivo studies demonstrating morphological alterations in certain cortical neurons after BF lesions. Although the demonstration of these effects in a highly simplified dissociated culture system lends support to the idea that the stimulatory signals are provided to cortical neurons directly by BFCNs, present results do not eliminate indirect effects. Possible direct mediators might include the neurotransmitter acetylcholine, other anterogradely transported trophic molecules (Corfas et al., 1995), or membrane-bound molecules. Possible indirect mediators could include other neurons or cortical glial cells. Present results, taken together with our previous study demonstrating survival- and growth-enhancing effects of cortical neurons on BFCNs (Ha et al., 1996a), suggest the existence of reciprocal interactions between BFCNs and certain cortical neurons that can be modeled in a simplified culture system. Because these effects appear to be maximal at culture ages corresponding to the period during which BFCNs grow into and innervate cortex, they may well be relevant to the development of BFCN-cortical projections in vivo. In addition, the present culture system may prove useful for studies relevant to Alzheimer's disease or other conditions in which there is degeneration of both BFCNs and SMI-32-immunoreactive cortical pyramidal neurons (Davies and Maloney, 1976; Whitehouse et al., 1982; Morrison et al., 1987; Hof et al., 1990; Cullen et al., 1997).

\section{REFERENCES}

Arendash GW, Millard WJ, Dunn AJ, Meyer EM (1987) Long-term neuropathological and neurochemical effects of nucleus basalis lesions in the rat. Science 238:952-956.

Baratta J, Ha DH, Weiss JH, Yu J, Robertson RT (1996) Cholinergic neurons from different subdivisions of the basal forebrain lack connectional specificity for cerebral cortical target sites in vitro. Dev Brain Res 97:143-147.

Bigl V, Woolf NJ, Butcher LL (1982) Cholinergic projections from the basal forebrain to frontal, parietal, temporal, occipital, and cingulate cortices: a combined fluorescent tracer and acetylcholinesterase analysis. Brain Res Bull 8:727-749.

Book AA, Wiley RG, Schweitzer JB (1992) Specificity of 192 IgGsaporin for NGF receptor-positive cholinergic basal forebrain neurons in the rat. Brain Res 590:350-355.

Book AA, Wiley RG, Schweitzer JB (1995) 192 IgG-saporin 2. Neuropathology in the rat brain. Acta Neuropathol (Berl) 89:519-526.
Calarco CA, Robertson RT (1995) Development of basal forebrain projections to visual cortex: DiI studies in rat. J Comp Neurol 354:608-626.

Chandler CE, Parsons LM, Hosang M, Shooter EM (1984) A monoclonal antibody modulates the interaction of nerve growth factor with PC12 cells. J Biol Chem 259:6882-6889.

Corfas G, Rosen KM, Aratake H, Krauss R, Fischbach GD (1995) Differential expression of ARIA isoforms in the rat brain. Neuron 14:103-115.

Crowley C, Spencer SD, Nishimura MC, Chen KS, Pitts-Meek S, Armanini MP, Ling LH, MacMahon SB, Shelton DL, Levinson AD, Phillips HS (1994) Mice lacking nerve growth factor display perinatal loss of sensory and sympathetic neurons yet develop basal forebrain cholinergic neurons. Cell 76:1001-1011.

Cullen KM, Halliday GM, Double KL, Brooks WS, Creasey H, Broe GA (1997) Cell loss in the nucleus basalis is related to regional cortical atrophy in Alzheimer's disease. Neuroscience 78:641-652.

Davies P, Maloney AJ (1976) Selective loss of central cholinergic neurons in Alzheimer's disease. Lancet 2:1403.

De Carlos JA, Schlaggar BL, O'Leary DDM (1995) Development of acetylcholinesterase-positive thalamic and basal forebrain afferents to embryonic rat neocortex. Exp Brain Res 104:385-401.

Dinopoulos A, Eadie LA, Dori I, Parnavelas JG (1989) The development of basal forebrain projections to the rat visual cortex. Exp Brain Res 76:563-571.

Divac I (1975) Magnocellular nuclei of the basal forebrain project to neocortex, brain stem, and olfactory bulb. Review of some functional correlates. Brain Res 93:385-398.

Eckenstein FP, Baughman RW, Quinn J (1988) An anatomical study of cholinergic innervation in the rat cerebral cortex. Neuroscience 25:457-474.

Gage FH, Wictorin K, Fischer W, Williams LR, Varon S, Bjorklund A (1986) Retrograde cell changes in the medial septum and diagonal band following fimbria-fornix transection: quantitative temporal analysis. Neuroscience 19:241-255.

Gargano N, Levi A, Alema S (1997) Modulation of nerve growth factor internalization by direct interaction between $\mathrm{p} 75$ and Trk A receptors. J Neurosci Res 50:1-12.

Gould E, Woolf NJ, Butcher LL (1991) Postnatal development of cholinergic neurons in the rat. I. Forebrain. Brain Res Bull 27:767-789.

Ha DH, Robertson RT, Ribak CE, Weiss JH (1996a) Cultured basal forebrain cholinergic neurons in contact with cortical cells display synapses, enhanced morphological features, and decreased dependence on nerve growth factor. J Comp Neurol 373:451-465.

Ha DH, Robertson RT, Weiss JH (1996b) Trophic interaction between basal forebrain neurons and cortical neurons in dissociated co-cultures. Soc Neurosci Abstr 22:744.

Hartikka J, Hefti F (1988) Development of septal cholinergic neurons in culture: plating density and glial cells modulate effects of NGF on survival, fiber growth, and expression of transmitter-specific enzymes. J Neurosci 8:2967-2985.

Hatanaka H, Tsukui H, Nihonmatsu I (1988) Developmental change in the nerve growth factor action from induction of choline acetyltransferase to promotion of cell survival in cultured basal forebrain cholinergic neurons from postnatal rats. Dev Brain Res 39:85-95.

Heckers S, Ohtake T, Wiley RG, Lappi DA, Geula C, Mesulam M (1994) Complete and selective cholinergic denervation of rat neocortex and hippocampus but not amygdala by an immunotoxin against the p75 NGF receptor. J Neurosci 14:1271-1289.

Hefti F (1986) Nerve growth factor promotes survival of septal cholinergic neurons after fimbrial transections. J Neurosci 6:2155-2162.

Hof PR, Morrison JH (1990) Quantitative analysis of a vulnerable subset of pyramidal neurons in Alzheimer's disease. II. Primary and secondary visual cortex. J Comp Neurol 301:55-64.

Hof PR, Cox K, Morrison JH (1990) Quantitative analysis of a vulnerable subset of pyramidal neurons in Alzheimer's disease. I. Superior frontal and inferior temporal cortex. J Comp Neurol 301:44-54.

Hohmann CF, Kwiterovich KK, Oster-Granite ML, Coyle JT (1991) Newborn basal forebrain lesions disrupt cortical cytodifferentiation as visualized by rapid Golgi staining. Cereb Cortex 1:143-157.

Houser CR (1990) Cholinergic synapses in the central nervous system: studies of the immunocytochemical localization of choline acetyltransferase. J Electron Microsc Tech 15:2-19.

Houser CR, Crawford GD, Salvaterra PM, Vaughn JE (1985) Immunocytochemical localization of choline acetyltransferase in rat cerebral 
cortex: a study of cholinergic neurons and synapses. J Comp Neurol 234:17-34.

Hsiang J, Heller A, Hoffmann PC, Mobley WC, Wainer BH (1989) The effects of nerve growth factor on the development of septal cholinergic neurons in reaggregate cell cultures. Neuroscience 29:209-223.

Koliatsos VE, Price DL, Gouras GK, Cayoutte MH, Burton LE, Winslow JW (1994) Highly selective effects of nerve growth factor, brainderived neurotrophic factor, and neurotrophin-3 on intact and injured basal forebrain magnocellular neurons. J Comp Neurol 343:247-262.

Mesulam M, Geula C (1991) Differential distribution of a neurofilament protein epitope in acetylcholinesterase-rich neurons of human cerebral cortex. Brain Res 544:169-173.

Morrison JH, Lewis DA, Campbell MJ, Huntley GW, Benson DL, Bouras C (1987) A monoclonal antibody to non-phosphorylated neurofilament protein marks the vulnerable cortical neurons in Alzheimer's disease. Brain Res 416:331-336.

Nonomura T, Nishio C, Lindsay RM, Hatanaka H (1995) Cultured basal forebrain cholinergic neurons from postnatal rats show both overlapping and non-overlapping responses to the neurotrophins. Brain Res 683:129-139.

Robertson RT, Gallardo KA, Claytor KJ, Ha DH, Ku K, Yu BP, Lauterborn JC, Wiley RG, Yu J, Gall CM, Leslie FM (1998) Neonatal treatment with $192 \mathrm{IgG}$-saporin produces long term forebrain cholinergic deficits and reduces dendritic branching and spine density of neocortical pyramidal neurons. Cereb Cortex, 8:142-155.

Rose K, Goldberg MP, Choi DW (1993) Cytotoxicity in murine cortical cell culture. In: In vitro biological methods (Tyson CA, Frazier JM, eds), pp 46-60. San Diego: Academic.

Roßner S, Härtig W, Schliebs R, Brückner G, Brauer K, Perez-Polo JR, Wiley RG, Bigl V (1995) 192 IgG-saporin immunotoxin-induced loss of cholinergic cells differentially activates microglia in rat basal forebrain nuclei. J Neurosci Res 41:335-346.
Sofroniew MV, Cooper JD, Svendsen CN, Crossman P, Ip NY, Lindsay RM, Zafra F, Lindholm D (1993) Atrophy but not death of adult septal cholinergic neurons after ablation of target capacity to produce mRNAs for NGF, BDNF, and NT3. J Neurosci 13:5263-5276.

Svendsen CN, Kew JN, Staley K, Sofroniew MV (1994) Death of developing septal cholinergic neurons following NGF withdrawal in vitro: protection by protein synthesis inhibition. J Neurosci 14:75-87.

Tago H, Kimura H, Maeda T (1986) Visualization of detailed acetylcholinesterase fiber and neuron staining in rat brain by a sensitive histochemical procedure. J Histochem Cytochem 34:1431-1438.

Taniuchi M, Johnson Jr EM (1985) Characterization of the binding properties and retrograde axonal transport of a monoclonal antibody directed against the rat nerve growth factor receptor. J Cell Biol 101:1100-1106.

Wainer BH, Mesulam MM, Mufson EJ, Saper CB (1984) Cortical projections arising from the basal forebrain: a study of cholinergic and non-cholinergic compounds employing combined retrograde tracing and immunohistochemical localization of choline acetyltransferase. Neuroscience 13:627-643.

Wellman CL, Sengelaub DR (1991) Cortical neuroanatomical correlates of behavioral deficits produced by lesion of the basal forebrain in rats. Behav Neural Biol 56:1-24.

Wellman CL, Sengelaub DR (1995) Alterations in dendritic morphology of frontal cortical neurons after basal forebrain lesions in adult and aged rats. Brain Res 669:48-58.

Whitehouse PJ, Price DL, Struble RG, Clark AW, Coyle JT, Delon MR (1982) Alzheimer's disease and senile dementia: loss of neurons in the basal forebrain. Science 215:1237-1239.

Wiley RG, Oeltmann TN, Lappi DA (1991) Immunolesioning: selective destruction of neurons using immunotoxin to rat NGF receptor. Brain Res 562:149-153. 